\title{
DISTOPÍA Y MASCULINIDAD HEGEMÓNICA. LECCIONES FEMINISTAS DESDE EL FIN DEL MUNDO
}

\author{
Santiago Karim Jatib Mejías \\ Universidad de La Laguna \\ alu0100893373@ull.edu.es
}

\section{RESUMEN}

La comunidad científica lleva décadas alertando sobre las consecuencias negativas esperables a futuro de los modelos de desarrollo implantados en todo el mundo siguiendo las pautas dictadas desde el ámbito occidental del globo. Sin embargo, estos análisis y proyecciones han obviado de forma notabilísima los aspectos de género de la convivencia que ponen también en riesgo la existencia humana sobre el planeta. En el presente ejercicio se tomará como referencia la producción cinematográfica en torno a la distopía para diagnosticar las disfuncionalidades existentes en términos de ordenación sexual/identitaria de las comunidades humanas y su asociación con escenarios de abismo civilizatorio.

Palabras Clave: estudios culturales, masculinidades, cinematografía, distopía, identidades de género.

\author{
HEGEMONIC MASCULINITY AND DYSTOPIA. \\ FEMINIST LESSONS FROM THE END OF THE WORLD
}

\section{Abstract}

Scientific community has been for decades giving alert about the negative outcome to be expected from the development models set up globally following the patterns dictated by the Western side of the world. Nevertheless, those analysis and projections have remarkably ignored the gender aspects of coexistence that endanger as well the human existence on the planet. This exercise will take as reference the cinematographic production regarding dystopia to diagnose the disfunctionalities that affect the sexual/identitary organization of human communities and their asociation with civilizatory abyss scenarios.

KEYWORDs: cultural studies, masculinities, cinematography, dystopia, gender identities.

DOI: https://doi.org/10.25145/j.atlantid.2021.12.12

Revista Atlántida, 12; diciembre 2021, pp. 241-260; ISSN: e-2530-853X 


\section{INTRODUCCIÓN}

\section{Planteamiento del problema y objetivos}

Durante las últimas cuatro décadas ${ }^{1}$, la industria cinematográfica ha ofrecido numerosas producciones en las que la posibilidad del fin de la especie humana, y del mundo como esta lo ha conocido hasta ahora, suponen el núcleo de los relatos propuestos. En el cine sobre distopías se concatenan narraciones sobre la destrucción masiva del planeta y su biodiversidad, conflictos bélicos, desastres ecológicos irreparables y derivas violentas del desarrollo científico/tecnológico que ponen en riesgo objetivo la posibilidad de supervivencia del ser humano sobre la Tierra.

Se partirá aquí de la hipótesis de que, en escenarios apocalítpicos o cuasiapocalípticos como los descritos desde las distopías cinematográficas, existen importantes factores de género de los que dar cuenta desde una aproximación analítica enmarcada en el amplio catálogo metodológico disponible en el haber de las ciencias sociales, y en concreto, poniendo en funcionamiento los instrumentos científicos provistos desde la tradición de estudios feministas.

El presente ejercicio tiene como objetivos:

1. Ofrecer una lectura sociológica que permita reflexionar sobre el estado presente de la experiencia comunitaria humana, así como sobre sus potenciales futuros, tomando como referencia las estructuras narrativas provistas desde el cine de masas ${ }^{2}$.

2. Indagar en las relaciones existentes entre el cine distópico, los escenarios adversos planteados desde este y las diversas categorías propias del análisis feminista tales como el sistema sexo/género, la construcción de las identidades masculinas, la división sexual del trabajo y otras que resultaren pertinentes.

3. Proponer una reflexión crítica en torno a aquellos elementos empíricos que constituyen la realidad social y política contemporánea y que pudieran relacionarse causalmente con futuribles contextos límite para la supervivencia de la especie.

4. Establecer un marco de análisis amplio que se sirva de diversos enfoques pertenecientes al ámbito de las ciencias sociales, para una aproximación holística al problema de estudio.

1 Atendiendo a Marzábal Albaina (2009), los ejemplos más representativos de cine distópico empiezan a darse a partir de finales de la década de los 70, con filmes como Mad Max (1979), Blade Runner (1982) o Brazil (1985). Si bien, fuera de lo audiovisual, pueden encontrarse con anterioridad poderosos relatos sobre escenarios futuros desalentadores: novelas como Un mundo feliz de Aldous Huxley (1932) o 1984 de George Orwell (1949) se adelantaron décadas a las críticas que posteriormente se actualizarían desde el cine.

2 Se hará referencia a producciones mayoritariamente procedentes de grandes estudios norteamericanos o británicos, con estructuras de distribución de alcance global. 
A la hora de escoger las herramientas de investigación desde las que guiar el procedimiento de análisis que aquí tendrá lugar (y atendiendo a la naturaleza de la problemática escogida y de los elementos desde los que se buscará dar cuenta de la misma -la producción cinematográfica en torno a la distopía, en este caso-), se optará por la adopción de un enfoque cualitativo/interpretativo, incluyendo esta rúbrica -siguiendo a Piergiorgio Corbetta- «todas las perspectivas teóricas para las que la realidad no puede ser sólo observada, sino que debe ser "interpretada"» (Corbetta, 2007).

Dentro de la lógica interpretativa escogida, se primará el análisis de diversas producciones audiovisuales, si bien la aproximación vendrá dada desde un marco teórico amplio que incluirá categorías tomadas de los estudios culturales y de cine, la extensa y diversa producción teórica de la tradición feminista, las perspectivas ecologistas y las aportaciones procedentes de la literatura.

Se han considerado fundamentales aquí las aportaciones específicas de autoras y autores como Carlos Duarte, Judith Halberstam, Pierre Bourdieu, Paula Iadevito o José María Fernández Calleja -entre otras y otros-, suponiendo ello una muestra de la diversidad de disciplinas y aproximaciones teóricas y científicas considerada necesaria para elaborar una lectura orientada a ser holística y comprensiva, en tanto la problemática abordada desde el presente trabajo es asimismo enormemente multidimensional y requiere de un abordaje dotado de la mayor amplitud posible.

La heterogeneidad de enfoques y aportaciones empleadas para el estudio tiene que ver precisamente con la naturaleza interpretativa del mismo: si bien se ha partido de una hipótesis o intuición más o menos clara, el marco teórico, la selección de las fuentes de información y la selección de perspectivas para su tratamiento han sido procesos que se han intercalado con el propio visionado de las películas, a fin de adaptarse a las también heterogéneas, cambiantes y diversas exigencias que el problema teórico y el objeto de estudio han ido manifestando a lo largo de la presente elaboración.

\section{DESARROLLO}

\section{Miradas incompletas Sobre el futuro. La laguna de GÉNero}

El final del s. Xx y las dos primeras décadas del s. Xxi han destacado por ser un periodo histórico relativamente breve en el que se han concentrado innumerables eventos de carácter dramático para la especie humana y los entornos sociales, económicos, políticos y medioambientales en los que esta se integra y de los que participa. Catástrofes medioambientales y ecológicas sin precedentes (normalmente vinculadas a la acción del ser humano), enfrentamientos bélicos con consecuencias desmesuradas para la habitabilidad de áreas enteras del globo terráqueo, crisis económicas sucesivas cuya dimensión supera a muchas de las previamente acaecidas en términos 
históricos y demás acontecimientos de naturaleza convulsa han redefinido la forma en la que la Humanidad existe sobre la Tierra y reflexiona sobre dicha existencia ${ }^{3}$.

El catálogo de sucesos históricos alarmantes durante los últimos decenios es en sí extenso y relevante como objeto de estudio. No obstante, la inmensa significación de los eventos en cuestión está lejos de agotarse en su análisis historiográfico específico, habiendo dado lugar, por el contrario, a profundos análisis y reflexiones sobre el potencial devenir de las sociedades humanas.

El pensamiento sobre el futuro, retomando el testigo de las reivindicaciones de mayo del 68, ha encendido las alarmas en torno a la viabilidad de una experiencia humana profundamente atravesada por la violencia, el consumo desproporcionado de recursos naturales o las desigualdades sociales, sirviéndose para ello de las más diversas formas de expresión, desde las más espontáneas (protestas, manifestaciones, festivales) hasta las más institucionales, como la formación de burocracias partidistas y complejos movimientos internacionales ${ }^{4}$.

En este sentido -y en una suerte de culminación reciente de la tendencia reflexiva señalada ${ }^{5}$, el Centro Superior de Investigaciones Científicas (CSIC) publicaba en un estudio del año $2008^{6}$ una serie de predicciones sobre posibles escenarios a los que la Humanidad habría de enfrentarse en el futuro próximo. El análisis en cuestión describía con notable precisión las consecuencias a futuro de atender o no a las necesidades de transición ecológica de los modelos productivos, de adoptar estrategias reactivas o proactivas en torno a la resolución de conflictos nacionales o internacionales, o del grado de multilateralismo o unilateralidad con que estos fuesen resueltos.

No obstante, a pesar de la pericia del equipo investigador involucrado en el trabajo en cuestión y la amplitud de la investigación llevada a cabo, la lectura del documento desde una perspectiva sociológica integral revelaría la ausencia de un aspecto nuclear desde el que dar cuenta del porvenir de la Humanidad e inmediatamente vinculado a los factores económicos, ecológicos y al modo de entender las relaciones internacionales, entre otros pilares de la experiencia comunitaria humana.

El factor de género brillaría por su ausencia en el esmerado estudio desarrollado desde el CSIC, evidenciándose con ello la inexistencia de una óptica feminista a la hora de llevar a cabo análisis sobre el estado presente y futurible de la experien-

${ }^{3}$ Una descripción detallada de los desarrollos históricos globales más recientes puede encontrarse en la obra de Francisco Veiga El desequilibrio como orden. Una historia de la posguerra fría, que, en su última edición, documenta los eventos acontecidos hasta el año 2014 (Veiga, 2015).

${ }^{4}$ Para un análisis minucioso de la evolución de los movimientos sociales nacidos de las revueltas parisinas del 68, consultar las aportaciones de Florent Marcellesi al respecto (Marcellesi, 2008).

${ }^{5}$ Que puede retrotraerse, en su arista institucional, hasta acontecimientos de calado internacional como la elaboración del Informe Brundtland, documento elaborado por representantes de diversos países y presentado ante la ONU en 1987, y en el que ya se ponía de manifiesto la dudosa sostenibilidad del modelo de desarrollo capitalista globalizado.

${ }^{6}$ Cambio global. Impacto de la actividad humana sobre el sistema Tierra (Duarte et al., 2008). 
cia humana, así como la gran laguna existente en materia de comprensión y diagnóstico de la misma debida a la invisibilización del género como clave analítica.

Así, aspectos como la revisión de la división sexual del trabajo, la valorización de las labores de cuidados y reproductivas, las cuestiones identitarias o la vinculación de las construcciones de sexo y género con el reparto efectivo del poder en las sociedades presentes y futuras quedarían, por tanto, completamente desplazados del marco de estudio, provocando importantes carencias a la hora de esbozar potenciales escenarios de futuro para la especie humana, y para definir los principales obstáculos que caracterizarían dichos escenarios o las formas de hacerles frente. El minucioso análisis realizado por el CSIC, considerado desde una perspectiva de género, sufriría por tanto de un notabilísimo sesgo cuya naturaleza, por otro lado, habría sido puesta de manifiesto por la tradición feminista en el conjunto de las ciencias a lo largo de la Historia?.

LA ALTERNATIVA CINEMATOGRÁFICA: EL GÉNERO DE LA DISTOPÍA COMO MARCO DE ANÁLISIS

Teniéndose en el presente ejercicio la proyección de escenarios futuros como elemento de gran interés sociológico (una vez que se considera que permite visibilizar desde una perspectiva más amplia muchas de las problemáticas y conflictos que aquejan a las sociedades actuales y, con ello, elaborar reflexiones que potencialmente pudiesen neutralizar las amenazas más graves a que estas se enfrentan $)^{8}$, y pensándose en el factor de género como un aspecto que necesariamente ha de estar presente en las miradas que se planteen respecto al porvenir de la especie humana, se recurrirá aquí a otra amplísima fuente de visiones al futuro dotada de enorme relevancia social y sociológica: la producción cinematográfica y, en concreto, al género de la distopía.

Rescatando la visión de Erreguerena Albateiro, la distopía sería «una advertencia de cómo puede llegar a ser el futuro de una sociedad si no transformamos o eliminamos las tendencias del presente» (2008, p. 556). La distopía, por tanto, sería una proyección de los escenarios hostiles a los que las comunidades humanas habrían de enfrentarse en caso de no resolver los conflictos que habitan en su interior y que amenazan su supervivencia. El concepto, atendiendo a la autora, se ela-

7 Siguiendo la exposición de Marta Jiménez Jaén, el feminismo como perspectiva de análisis surgiría precisamente como reacción ante el histórico sesgo androcéntrico de la cultura y el conocimiento occidental, con la finalidad de poner dicha desigualdad de manifiesto y contribuir a corregirla (Jiménez Jaén, 2011).

${ }^{8}$ En palabras de Gaston Berger, "Prever una catástrofe es condicional: es prever lo que ocurriría si no hacemos nada por cambiar el curso de las cosas, y no lo que ocurrirá de todas maneras. Ver un átomo lo cambia, mirar un hombre lo transforma, ver el futuro lo cambia todo» (citado en E. Albaitero, 2008). 
boraría en contraposición al de utopía ${ }^{9}$, que haría referencia a un hipotético estado futuro ideal de las cosas en el que la Humanidad -o comunidades concretas dentro de esta- habría alcanzado el máximo grado de optimización de su experiencia colectiva, viviendo por tanto desprovista de conflictos sustanciales.

Siguiendo su planteamiento, «La palabra distopía es el antónimo de utopía. La palabra utopía significa etimológicamente "no lugar"; es una ficción de una sociedad inexistente. Por su parte, distopía significa lo "no deseable" (2008, p. 560).

Las elaboraciones audiovisuales en torno a la distopía serían, atendiendo a la visión analítica que aquí se busca proyectar, un recurso para el estudio desde el que localizar pistas sobre los sentidos comunes que en las últimas décadas (y en términos acumulativos, históricamente $)^{10}$ se han venido manejando en torno a los conceptos de sexo y género, así como sobre las prácticas en que estos se concretan y a través de las que evolucionan.

\section{Cinematografía y feminismo. ¿Por Qué el Cine?}

La ausencia de una perspectiva feminista en las proyecciones de futuro realizadas (en este caso, por el CSIC) lleva a la búsqueda de respuestas (así como a la propia formulación de las preguntas) a través del cine. La elección de este nicho de producción cultural no es, sin embargo, accidental.

Siguiendo a Paula Iadevito (2014), el cine constituiría un espacio de generación de discursos en torno a la realidad y, con ello, un lugar privilegiado para el estudio de procesos históricos y sociológicos. La autora apunta, así, a que «el cine constituye una fuente primaria de indagación acertada en el campo de la investigación social para leer analíticamente e interpretar los procesos identitarios y de subjetivación generizada», concretando cómo «la perspectiva de género en el análisis fílmico resulta productiva para comprender la relación entre mujeres y hombres a través de la historia e identificar los mecanismos de reproducción y cuestionamiento de las representaciones culturales y simbólicas hegemónica» (p. 214).

La aportación de Iadevito se aproxima a la intuición que guía el presente ejercicio: en sus palabras, «las narrativas del cine configuran espacios de significación que abren dimensiones ideológicas desde las cuales es posible observar las modali-

9 Si bien la utopía como concepto y género literario tiene su mayor referente (y su evento inaugural) en la Utopia de Tomás Moro (publicada en 1516), su tradición se expande enormemente a posteriori, pudiéndose citar como algunos referentes de la misma las obras Walden de Henri David Thoreau o la casi homónima (y más cercana a la perspectiva sociológica que aquí se busca desarrollar) Walden 2, de Burrhus Frederic Skinner.

${ }^{10}$ Se parte de la suposición de que las prácticas en torno al género y al sexo resultan de la cristalización de procesos históricos que repercuten sobre los sujetos, y cuyos efectos tienden a ser reproducidos por estos últimos. En este sentido, puede decirse que se parte de una concepción ciertamente bourdiana de las prácticas sociales. 
dades en que las marcas identitarias y de género instituyen las redes culturales de una sociedad» (2008, p. 214).

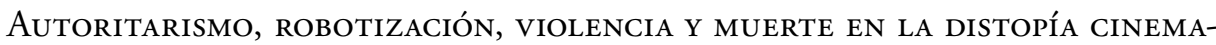
TOGRÁFICA. CRÍtiCAS DESDE LOS ESTUdios DE GÉNERO

Si bien el informe sobre el cambio global elaborado por el CSIC presentaba las lagunas analíticas que se han señalado en líneas anteriores, el trabajo coordinado por Carlos Duarte también apuntó a una serie de fenómenos que suponen en efecto una gran amenaza para la Humanidad en su conjunto: el surgimiento de nacionalismos excluyentes y violentos, la regionalización y fragmentación del mundo en una tendencia hacia el abandono del multilateralismo y la redefinición de la «seguridad nacional» en términos autoritarios y racistas. El cine distópico ha dado buena cuenta de esta problemática en diversas producciones ${ }^{11}$, uniéndola en sus narrativas a otra también muy frecuente en los escenarios abordados: la tecnología y sus hipotéticas derivas amenazantes, normalmente asociadas también a la violencia ${ }^{12}$.

Será en estas coordenadas narrativas en las que se desenvuelvan los relatos de filmes como Children of Men (2006, Alfonso Cuarón), Terminator I(James Cameron, 1984) y Terminator II (James Cameron, 1991), The Matrix y The Matrix Reloaded (Lana y Lilly Wachowski ${ }^{13}, 1999$ y 2003); o capítulos de la serie Black Mirror como «Men against fire» (2017, Jakob Verbruggen) y «Metalhead» (2017, David Slade), que servirán como materia prima para la elaboración del análisis que aquí se plantea.

Si bien estas producciones poseen sus desarrollos narrativos específicos, existe un mínimo común denominador en ellas que alude a un hipotético futuro a corto/medio plazo en el que las claves organizativas -o aspiracionales- de las sociedades occidentales (la democracia representativa, el Estado de Derecho, la defensa de los Derechos Humanos):

${ }^{11}$ Para una revisión sucinta de la producción cinematográfica sobre distopía, consultar el siguiente artículo del portal especializado en cine Espinof: https://www.espinof.com/otros/21-peliculas-distopicas-alucinantes-que-todo-fan-de-blade-runner-ya-deberia-haber-visto.

${ }^{12}$ Es notable el hecho de que no solo el cine, sino gran parte de la producción audiovisual en su conjunto se encuentre en las últimas décadas reproduciendo discursos cargados de un gran contingente de violencia. A este respecto, resultan fundamentales las aportaciones de Alessandro Gabbiadini y sus compañeros en torno a la presencia de la violencia en los videojuegos, sus implicaciones de género y sus efectos sobre la construcción de la identidad (Gabbiadini et al., 2013 y 2016).

${ }_{13}$ Las hermanas Wachowski, para el momento del estreno de las dos primeras entregas de Matrix, se identificaban como hombres cisgénero (Larry y Andy Wachowski). Años después, experimentarían procesos de transformación identitaria que les llevarían a identificarse como mujeres trans. Aquí serán referenciadas a través de sus nuevas identidades, si bien entrel@s seguidor@s de la saga The Matrix este cambio no siempre es conocido y en ocasiones se las sigue asociando con sus construcciones identitarias masculinas. 
a) han sido desplazadas en favor de mecanismos autoritarios desde los que arbitrar la convivencia en contexto de grandes tensiones sociales y políticas

b) directamente han desaparecido debido a la instauración de un nuevo orden político en el que el desarrollo tecnológico ha culminado en la hegemonía de las máquinas, reduciendo a la especie humana a un agente cuya máxima vocación ha pasado a ser la supervivencia ante entidades tecnológicas que buscan exterminarla.

El primer escenario es el reflejado más nítidamente en Children of Men (2006) y "Men against fire» (2016), el filme del mexicano Alfonso Cuarón y el capítulo de Black Mirror (serie de HBO), respectivamente.

Cuarón describe un futuro cercano (la película fue estrenada en 2006 y se ambienta en 2027) en el que las tensiones sociales, políticas, climáticas y demográficas ${ }^{14}$ han terminado deteriorando al límite el funcionamiento de los Estados-nación de todo el mundo, quedando Gran Bretańa como último baluarte del poder centralizado estatal, país en el que son aplicadas sistemáticamente políticas enormemente represivas, violentas y racistas a fin de garantizar la pervivencia del statu $q u o$. Por su parte, «Men against fire» relata la experiencia de una tropa de soldados que se dedica a perseguir y asesinar a un grupo de seres conocidos como cucarachas (en inglés, roaches), para lo que reciben entrenamiento y una equipación tecnológica muy sofisticada consistente en un software intracerebral que se encarga de regular y organizar el funcionamiento de todos sus sentidos ${ }^{15}$ y de gestionar y procesar la información que estos reciben. Dichas «cucarachas», como descubre eventualmente Stripe - protagonista del capítulo-, terminan siendo grupos de ciudadanos marginados ${ }^{16}$ que los ojos de los soldados perciben (debido al software militar implantado en sus cerebros) como una especie de monstruos a quienes asesinar indiscriminadamente, de forma casi lúdica ${ }^{17}$.

${ }^{14}$ El núcleo argumental de la película reside en que toda la población del planeta, aparentemente, se ha quedado estéril.

${ }^{15}$ El asunto de la neurotecnología y de las problemáticas éticas, filosóficas y sociológicas asociadas a esta constituyen una constante en los relatos expuestos a través de Black Mirror, destacando en este sentido capítulos como «Arkangel», «USS Callister»o «Black Museum» (en este último se realiza, además, una soberbia crítica al racismo estructural presente en Estados Unidos). La relevancia de la gestión tecnológica de lo neuronal constituye ya actualmente un asunto de primer orden, evidenciándose ello en procesos como la actual tramitación gubernamental en Espańa de una «Carta de los Derechos Digitales», en cuyo primer borrador ya se alude explícitamente a la existencia de «neuroderechos».

${ }^{16} \mathrm{Si}$ bien en el capítulo no se especifica cuál es el motivo de dicha marginación, la audiencia ha interpretado que podría tratarse - al igual que en el filme de Cuarón- de personas migrantes. En este sentido, es relevante señalar la lectura que Owen Jones, destacado escritor y periodista británico, realizó al respecto, en la que identificó directamente el trato dado por los soldados a las "cucarachas» en el episodio de Black Mirror con el tratamiento dado por los gobiernos europeos a las personas refugiadas (https://www.theguardian.com/commentisfree/2016/oct/28/black-mirror-empathy-shared-humanity).

${ }_{17}$ Parece imposible no recordar frente a este relato los sucesos acontecidos durante el Holocausto nazi a principios del s. xx, y la normalidad burocrática con que el funcionariado bajo 
En ambas producciones se pone de manifiesto la hostilidad de los más que probables futuros (y en algunos casos, ya materializados presentes) ${ }^{18}$ de aquellas construcciones nacionales basadas en el odio y el miedo hacia quienes se entienden como externos a las mismas ${ }^{19}$.

En este sentido, se asiste en los filmes a una gestión del conflicto y la confrontación que entronca con lo que Fernández Calleja convino en denominar «una forma deliberadamente masculina de hacer política» en sus estudios sobre los factores de género que mediaron en el estallido y desarrollo de la Primera Guerra Mundial. Siguiendo al autor (Fernández Calleja, 2014), «La Gran Guerra fue un despliegue de masculinidad, de valores masculinos, presentados como superiores y distintos. Valores masculinos que se mostraban inmutables ante las consecuencias mortales de la guerra, ante el destrozo de vidas que esta supone. Mayreder (1981: 97), recrimina esa insensibilidad masculina: [Ser lo más viril posible...] es la verdadera distinción a los ojos de los hombres. Son insensibles a la brutalidad de la derrota o a la pura injusticia de un acto a condición de que coincida con el canon tradicional de masculinidad».

Esta visión sobre la relación entre lo que Mayreder (Fernández Calleja, 2014) señala como "canon tradicional de masculinidad» y los elementos de violencia y agresividad total vinculados a la práctica de la guerra (y que se presentaron en su máxima expresión conocida hasta entonces durante la Primera Guerra Mundial, siguiendo a los autores) encuentra un fuerte sustento teórico en las elaboraciones de Raewyn Connell (Connell, 2003) en torno a las masculinidades, la diversidad de formas que estas pueden adoptar y los términos en que se organizan de forma jerárquica en torno a una modalidad de masculinidad que es la hegemónica y subordina a las demás (Connell, 2003).

La vinculación entre las formas hegemónicas de masculinidad ${ }^{20}$ (ligadas a la agresividad, a la violencia, a la competición -es decir esto, a todos los elementos comportamental/normativos opuestos a lo que se considera «femenino» desde dicha

el régimen nazi ejecutó las directrices ligadas a la «solución final»contra judí@s, homosexuales, gitan@s y demás minorías, habiendo sido teorizado dicho fenómeno por Hannah Arendt mediante la expresión de «la banalidad del mal».

${ }_{18}$ Alfonso Cuarón relata en su distopía cómo las personas que inmigran de forma ilegal son tratadas como criminales y enjauladas colectivamente. Dicha predicción, realizada en su ejercicio cinematográfico de 2006, terminó convirtiéndose en una realidad a la que asistió el mundo entero cuando Donald Trump decidió enjaular a familias enteras de migrantes centroamericanos a partir del año 2018 (https://www.bbc.com/mundo/noticias-internacional-44528183).

19 A este respecto, es oportuno rescatar las aportaciones de Ernesto Laclau en La razón populista en torno a los procesos de construcción nacional y a los mecanismos discursivos de exclusión sobre los que estos se sustentan. Atendiendo al autor, dichos procesos no tendrían necesariamente que guardar relación con relatos racistas, misóginos o lgbtifóbicos, sino que, como habrían puesto de manifiesto los "populismos» progresistas a lo largo del mundo, podrían estructurarse a partir de discursos comprometidos con la defensa de los derechos humanos y las reivindicaciones herederas de la filosofía política republicana (Laclau, 2005).

${ }^{20}$ Connell describe la masculinidad hegemónica como «la configuración de la práctica de género que incorpora la respuesta aceptada, en un momento específico, al problema de la legitimidad 
masculinidad-) y las formas de política basadas en la confrontación, la guerra y sus prácticas aparece aún más clara si se atiende a las reflexiones de Connell sobre los lazos que unen a dichas formas de masculinidad y elementos como la posesión de armas (2003, pp. 285-286). La autora apunta a la asociación simbólica de las armas (herramienta de dominación y poder) con el pene dentro de una configuración concreta de la masculinidad que se sustenta, precisamente, sobre la dominación y el poder.

El elemento armamentísico conecta directamente con el segundo tipo de escenario descrito en la filmografía analizada: aquel en que el desarrollo tecnológico ha coadyuvado en su última expresión a la amenaza de extinción de la especie humana por parte de máquinas programadas para desconocer cualquier consideración vinculada a elementos de empatía o derechos humanos. Es esta línea narrativa la que sirve de núcleo para el desarrollo de la saga Matrix, Terminator o "Metalhead» (episodio de Black Mirror). En dichos filmes, una mermadísima especie humana sobrevive contra diversas formas de tiranías tecnológicas que prácticamente han ganado la batalla contra esta, y que o bien buscan exterminarla definitivamente, $o$ bien pretenden mantenerla viva con el objetivo de esclavizarla o aprovechar su energía como combustible ${ }^{21}$.

Aplicando este marco de análisis a las producciones audiovisuales aquí estudiadas, el factor de género ( $y$, en concreto, la continuidad a futuro de sociedades articuladas en torno a criterios masculinos hegemónicos) se convierte en un mirador desde el cual se resignifica la práctica totalidad de las proyecciones realizadas desde la distopía en el cine. ¿Qué conclusiones arroja sobre la sostenibilidad del actual statu quo del sistema sexo/género el visionado de sagas como The Matrix o Terminator, en las que las armas de fuego, la sangre y la muerte constituyen prácticamente el núcleo de la narrativa sobre el futuro que se está esbozando? ¿Qué mensaje se decanta de "Metalhead» en Black Mirror, capítulo en el que las máquinas (creadas por seres humanos) funcionan como meros instrumentos de exterminio de todo lo que se resista a convertirse a este marco epistémico de violencia ilimitada?

Si bien es evidente la claridad que el análisis de género ya adquiere a estas alturas del ejercicio, es pertinente añadir un importante matiz: los conflictos que aquí buscan ser explicados en términos de prácticas hegemónicamente masculinas no guardan relación obligatoriamente con identidades de hombres cisgénero (si bien son estos quienes colonizan la mayor parte de los desarrollos narrativos en las producciones estudiadas). Personajes de mujeres cisgénero ${ }^{22}$ como Raiman (soldado

del patriarcado, lo que garantiza (o se considera que garantiza) la posición dominante de los hombres y la subordinación de las mujeres» (p. 117).

${ }^{21}$ Parece pertinente recordar en este punto las críticas realizadas por autores como Horkheimer o Adorno (y la Escuela de Frankfurt, en general) en torno a las ideas de progreso, desarrollo y razón propias del programa axiológico ilustrado, que ya habrían tenido una deriva nefasta resultante en las dos guerras mundiales del s. xx (Adorno y Horkheimer, 2007), y que, atendiendo a los filmes aquí estudiados, seguirían provocando enormes disfuncionalidades.

22 Si bien aquí se circunscribe el estudio a una serie de personajes específicos, el papel de las mujeres en el cine de distopía ha sido objeto de un estudio minucioso a través de diversos ejerci- 
compañera de Stripe, el protagonista de «Men against fire») o Trinity (lideresa de la resistencia humana en la saga The Matrix) ponen de manifiesto que el ejercicio de un esquema comportamental belicista y violento (que aquí se entiende precisamente como consecuencia de sociedades articuladas históricamente desde dichos parámetros, y, por tanto, como requisito para la supervivencia y reproducción de los sujetos en el marco de estas) comporta una forma de estar en el mundo que corresponde a identidades construidas en torno a lo masculino, que no exclusivamente a los varones cisgénero ${ }^{23}$, sino también a mujeres cisgénero y a varones transgénero ${ }^{24}$.

En este sentido, cobran gran relevancia las reflexiones de Judith Halberstam sobre lo que ella denomina masculinidades femeninas, y que, curiosamente, ejemplifica también a través de personajes femeninos del cine distópico. Halberstam documenta los caracteres subjetivos manifiestamente masculinos (agresividad, fuerza física, rudeza) presentes en Sara Connor (interpretada por Linda Hamilton), protagonista de la saga Terminator, o en Ellen Ripley (interpretada por Sigourney Weaver) en Aliens apuntando a que la adopción de estos rasgos asociados a las masculinidades hegemónicas formaría parte de una estrategia de supervivencia dentro de un marco sociopolítico en que dichos rasgos resultan clave para ser reconocid@ y legitimad@ ${ }^{25}$ (Halberstam, 2008), que ya operan en condiciones históricas ordinarias, y que, a la luz de dichos filmes, se exacerbarían en situaciones límite.

La lectura realizada por la autora es más que relevante a la luz de la visión que aquí se pretende elaborar: Sarah Connor, Raiman, Trinity, Katniss (Los juegos del hambre), Shaileen (Divergente) son personajes femeninos que gestionan sus iden-

cios académicos. Sirvan como referencia, por ejemplo, las aportaciones de Riestra-Camacho (2014) y Menéndez Menéndez y Fernández Morales (2015) en torno al liderazgo femenino en los filmes Divergente (2014, Neil Burguer) y Los juegos del hambre (2012, Gary Ross).

23 Se entiende por "cisgénero» a aquellas personas cuya identidad de género autopercibida coincide con aquella que les fue asignada al nacer (es decir, aquellas personas que no son trans).

${ }^{24}$ Esta concepción de las identidades de género como elementos contingentes y arbitrarios -en tanto no «naturales»- enlaza con la aportación de Judith Butler en relación con la problemática en cuestión. La autora señala que «El género es la estilización repetida del cuerpo, una sucesión de acciones repetidas -dentro de un marco regulador muy estricto- que se inmoviliza con el tiempo para crear la apariencia de sustancia, de una especie natural de ser» (Butler, 1990).

25 En palabras de la autora, «Podríamos abordar estas preguntas pensando en los efectos sociales y culturales de una escritura del género invertida. En otras palabras, ¡cuáles son las implicaciones de la feminidad masculina y de la masculinidad femenina? Podríamos imaginar que el más leve toque de feminidad mancillaría o rebajaría el valor social del varón, mientras que todas las formas masculinas adoptadas por mujeres producirían una elevación del estatus» (2008, p. 73). Halberstam alerta, no obstante, de que la cuota de poder que una mujer puede obtener mediante su asimilación a la masculinidad hegemónica peligraría en caso de que esta no se mantuviese dentro del mandato de la heterosexualidad. A su juicio, la combinación de una masculinidad femenina con una direccionalidad del deseo no heteronormativa pondría en peligro la posibilidad de legitimación social ligada al primero de los factores en cuestión. La razón, según la autora: una mujer masculina y queer se consideraría excesivamente masculina (p. 74). Esto lleva a pensar en cómo una mujer «excesivamente masculina» supondría una amenaza de hecho para el reparto de los beneficios simbólicos ligados a la masculinidad, aparentemente escasos e históricamente concebidos como privilegio del hombre cisgénero heterosexual. 
tidades de género en términos de supervivencia, empoderándose como mujeres pero dentro de los términos acotados por un régimen de masculinidad hegemónica que, articulado desde la violencia, la LGTBIQfobia, la misoginia y la erosión del entorno natural ${ }^{26}$, lleva a la humanidad a estados que fronterizan con su extinción, y cuya proximidad se demuestra cada vez más cercana. El hecho de ser un «hombre» o una «mujer», por tanto, quedaría en un segundo plano en tanto la práctica comportamental no se situase en coordenadas alternativas a las dominantes ${ }^{27}$.

REPRODUCCIÓN, CUIDADOS, AFECTO. RESQUiCIOS PARA UNA ALTERNATIVA FEMINISTA EN EL FIN DEL MUNDO

Vistas las claves narrativas presentes en el cine distópico, cabe preguntarse cuáles son los resortes activados por sus personajes para resarcir a la Humanidad de su deriva apocalíptica y devolverla a estados existenciales en que la supervivencia pudiese darse por sentada, y el recorrido de la especie humana siguiera produciéndose con cierta estabilidad.

Numerosos son los relatos fílmicos de las últimas décadas que apelan a la aparición de líderes o lideresas de naturaleza sobrehumana, a heroicidades místicas que responden a complejas tramas narrativas normalmente asociadas a construcciones de sentido cercanas al pensamiento mágico y metafísico. Así, los héroes y heroínas de los universos Marvel y DC, por ejemplo, destacan por presentarse como sujetos andromórficos, si bien prácticamente sobrenaturales.

Dotados de habilidades y superpoderes completamente ajenos a la experiencia humana ${ }^{28}$, personajes como Spiderman, Aquaman, Ironman, Thor ${ }^{29}$, etc., batallan, amparados en sus rasgos sobrehumanos, contra sujetos y fuerzas abyectas que amenazan a poblaciones totalmente indefensas y dependientes de la labor mesiánica, mágica y completamente individualizada de dichos superhéroes.

Dentro de esta tónica narrativa, Neo, protagonista de The Matrix, se lanza a batallar contra una red de máquinas que cultivan a seres humanos en una suerte de invernaderos para alimentarse de su energía orgánica. La trama discurre con cierta coherencia hasta que el protagonista, al borde de la muerte, logra evadir la muerte mediante mecanismos notablemente inciertos: durante el punto álgido de la primera

${ }^{26}$ La relación entre el patriarcado y el deterioro ambiental ha sido teorizada ampliamente desde la perspectiva ecofeminista (Castells, 1998).

27 Como apuntan Menéndez Menéndez y Fernández Morales (2015): «Así, Katniss podría responder a la propuesta de Savater: "la virilidad del héroe es esencial, aunque su sexo puede ser masculino o femenino"» (p. 98).

${ }^{28}$ La construcción de estos personajes aparece, desde una perspectiva weberiana, como una actualización de la modalidad carismática de la legitimidad.

${ }^{29}$ No parece casualidad, desde el marco de análisis aquí planteado, la tendencia a que los superhéroes -aquellos capaces de salvar a la Humanidad de la desaparición-sean mayoritariamente hombres cisgénero. 
entrega de la saga, Neo está ciertamente condenado a la derrota tras una ardua batalla con sus enemigos; no obstante, logra revivir de forma milagrosa y sobreponerse a las máquinas tras recibir un beso de Trinity, su amada.

Del mismo modo que un beso salva a Neo, en «Men Against Fire», Stripe y el resto de soldados (hombres y mujeres) reciben todas las noches sesiones de sueño programadas (a través del software implantado en sus cerebros) en las que el erotismo, el contacto físico y la proximidad con sus seres amados son los protagonistas. Dichas sesiones de sueño les permiten sobreponerse a un interminable caudal de estímulos negativos, procedentes de jornadas de entrenamiento militar y exterminio de seres humanos, a que se ven abocados diariamente.

En Metalhead, el escenario generalizado de muerte y continua alerta ante los perros (animales electrónicos diseñados para aniquilar toda vida humana que detecten) encuentra su única cadencia en la relación de camaradería entre Bella y sus dos compañeros, que irrumpen en un almacén abandonado buscando juguetes que llevar a quien se entiende que es un niño de su grupo que se enfrenta a los últimos días de su vida.

En Children of Men, una Gran Bretaña -y un mundo- caracterizados por el trato inhumano a las personas migrantes, por la contaminación y la hostilidad como modus operandi de las instituciones respecto a la población civil, quedan completamente paralizados ante el milagro del primer nacimiento en el globo tras 18 años de infertilidad generalizada ${ }^{30}$.

En primera instancia, el recurso al elemento afectivo/romántico/erótico en estos filmes podría interpretarse, efectivamente, como un necesario respiro para la audiencia, cuya atención e interés sería difícilmente sostenible de no permitir pequeños momentos para la gratificación emocional ante un contexto visual mayoritariamente desincentivador. No obstante, el beso entre Neo y Trinity, los sueños eróticos de Stripe y sus compañer@s, la caja de peluches de Metalhead o el nacimiento del hijo de Kee arrojan una importante pista sobre la relevancia del feminismo como clave para entender los diagnósticos realizados desde el género de la distopía y construir una alternativa. Estos breves espacios para la experiencia del contacto humano generativo, del amor en el amplio sentido del concepto (descrito acertadamente por Pierre Bourdieu como una suspensión de la violencia -Bourdieu, 2000-), constituyen un momento de ruptura dentro de relatos totalmente colonizados por la agresividad, sin contemplaciones hacia otros aspectos de la experiencia humana que se separen de dicho belicismo o lo desplacen.

Volviendo a las coordenadas teóricas de Raewyn Connell (en las que la idea de masculinidad hegemónica funciona, en cierto modo, como un nuevo espacio de impugnación y como factor explicativo de importantes problemáticas), las escenas

30 Es especialmente gráfica en este sentido la «suspensión de la violencia» de la que hablaba Bourdieu en La dominación masculina durante la escena final del filme de Cuarón, en la que un enfrentamiento bélico entre tropas estatales y la insurgencia ciudadana se detiene, súbitamente, al ver ambos bandos que Theo y Kee (protagonistas del largometraje) llevan en sus manos al hijo de Kee. 
señaladas podrían entenderse como una fractura efectiva de los términos en que se viene pretendiendo librar la batalla por la continuidad de la especie humana. Es decir: un modelo basado en la confrontación, en el ejercicio de la fuerza física, en la escalada violenta; un modelo deliberadamente masculino de organizar la convivencia dentro de las comunidades tendría, al fin y al cabo, «lagunas» en que los valores preconizados desde la teoría y la práctica feminista encontrarían su lugar, siendo dichas grietas en el modelo, paradójicamente, la condición de posibilidad para la supervivencia humana, como ponen de manifiesto los propios relatos desarrollados dentro de las producciones analizadas.

Así, la pregunta sobre los medios para la continuidad de la especie humana encontraría una respuesta factible, aparentemente, no en la superheroización ${ }^{31}$ de los personajes (elemento que no solo no rompe con la violencia, aumentando, por el contrario, su espectacularización), sino en los elementos netamente humanos -aparentemente residuales y secundarios, aun siendo los que permiten que los relatos se desarrollen y culminen de forma alentadora y con cierta verosimilitud ${ }^{32}$ - ya señalados, y que guardarían una fuerte relación con las propuestas y alternativas ofrecidas históricamente desde la tradición del pensamiento feminista.

Respecto a los aspectos afectivos, corporales y emocionales de la vida humana, el análisis feminista ha planteado históricamente cómo, debido a la división sexual del trabajo, estos se han visto desplazados simbólica y objetivamente a la esfera de la vida reservada a las mujeres en el marco de las sociedades patriarcales ${ }^{33}$. Si bien no exenta de problematización teórica y política dentro del propio movi-

31 Este término (utilizado por Mihai y Alin Răuțoiu (2016) en sus estudios sobre la industria del cómic) es empleado en el presente ejercicio para dar cuenta del fenómeno por el cual algunos de los personajes aquí estudiados reciben rasgos y dotaciones sobrehumanas que funcionan como ventajas frente a sus oponentes. Desde la lectura que aquí se hace de este proceso, se lo considera como un recurso narrativo necesario en contextos en que la hipermasculinización se convierte en un callejón sin salida, y es necesario desatascar la confrontación ampliando los términos en que se produce en lugar de recurrir a vías alternativas para la resolución de los conflictos relatados.

32 En su estudio sobre la estructura narrativa de la serie El cuento de la criada-otra producción audiovisual reciente ambientada en un hipotético futuro distópico marcado por una crisis demográfica y de natalidad-, Victoria Hernández Ruiz pone de manifiesto cómo aquellos relatos en que están ausentes aspectos nucleares de la experiencia humana como el afecto serían, desde la perspectiva del análisis literario, inverosímiles. De entre las observaciones de la autora destaca la siguiente síntesis: «Se ha avanzado en los anteriores epígrafes, gracias a distintas teorías literarias, que un mundo ficcional sin amor no es verosímil, dadas las condiciones de credibilidad para el lector y de coherencia interna de los elementos ficcionales, por un lado; y la necesidad de que el mundo posible posea estructuras universales que permitan a los particulares posibles interaccionar entre sí y con la estructura de orden general que sustenta dicho mundo. Se ha concluido también que el amor, como relación social intrínseca, configura como propiedad necesaria y esencial la estructura del mundo» (Hernández Ruiz, 2019).

$33 \mathrm{Si}$ bien esta exposición ha sido desarrollada por gran parte del feminismo del s. xx (Amorós, 1994), puede encontrarse un ejemplo particularmente minucioso en las aportaciones de Nuria Sánchez Mirá en torno a la naturaleza estructural de la división sexual del trabajo y a su evolución histórica (Sánchez Mirá, 2016). 
miento feminista ${ }^{34}$, la toma de conciencia sobre dicha desigualdad estructural ha allanado el camino para imaginar configuraciones del mundo que no tengan que ver con el actual statu quo, articulado en función de los criterios de la masculinidad hegemónica (y de cuyas características se ha nutrido la imaginación cinematográfica para esbozar potenciales futuros distópicos), sino con las experiencias que se han gestado dentro de la parte del mundo reservada para todas las identidades que han quedado excluidas, y, en concreto, dentro de la realidad de las tareas reproductivas y de cuidados, ejercidas por mujeres.

La intuición sobre la posibilidad de una manera alternativa de organizar la convivencia humana, planteada explícitamente en clave de género, encuentra un ejemplo particularmente brillante -y precoz ${ }^{35}$ - en la obra de Virginia Woolf, quien en Tres Guineas (1938) ya diagnosticaba la urgencia de un cambio a este respecto. La autora británica, ante la pregunta sobre qué podían aportar las hijas de los hombres instruidos para evitar el estallido de la guerra, ponía de manifiesto la inoperancia de la institucionalidad sociopolítica establecida para el logro de dicho fin, planteando la necesidad de repensar desde el feminismo cuáles eran las condiciones históricas que posibilitaban la amenaza continua de la guerra (Woolf, 2017) ${ }^{36}$.

El planteamiento de Woolf se produjo en un marco temporal en que ya tenía sentido, y vino sucedido por una serie de acontecimientos y procesos históri$\cos ^{37}$ que lo convierten en la actualidad en una formulación pionera y acompañada

${ }^{34}$ Como señala Celia Amorós, existe una tendencia dentro del movimiento feminista -el feminismo de la diferencia- que reivindica como propios y legítimos lo que considera como rasgos identitarios esenciales de las mujeres, entre los cuales se encontraría la maternidad y numerosos aspectos sociológico/psicológicos vinculados a ella (Amorós, 1994).

35 Tres Guineas, de Virgina Woolf, data de 1938, fecha previa al estallido de la II Guerra Mundial, al desarrollo de los Estados del bienestar y a la crítica feminista de estos. Del mismo modo, Tres Guineas se adelantó al desarrollo académico de los estudios de género en general y al de las masculinidades en concreto, lo que la ha convertido en una obra visionaria celebrada y homenajeada posteriormente (Pierre Bourdieu, por ejemplo, la reivindica como fuente de gran relevancia en su estudio La dominación masculina [Bourdieu, 2000]).

${ }^{36}$ En su exposición, Woolf sostenía: «¿No es esto suficiente? ¿'Tenemos que buscar más hechos de la historia y la biografía para demostrar que debe abandonarse todo intento de influir en los jóvenes contra la guerra a través de la educación que reciben en las universidades? ¿Acaso no ha quedado demostrado que la educación, la mejor educación del mundo, no enseña a aborrecer la fuerza, sino a utilizarla?: No ha quedado demostrado que la educación, lejos de enseñar a los instruidos la generosidad y la magnanimidad, crea en ellos por el contrario tales ansias de conservar sus posesiones, "la grandeza y el poder" de que habla el poeta, en sus propias manos que no emplearán la fuerza, sino medios más sutiles que la fuerza cuando se les pida que las compartan? ¿Y acaso la fuerza y el deseo de posesión no están íntimamente relacionados con la guerra? ¿De qué sirve pues la educación universitaria a la hora de influir en la gente para evitar la guerra?» (Woolf, 2017).

${ }_{37}$ Entre estos caben ser destacados: la II Guerra Mundial, la Guerra Fría, la caída del «socialismo real», el desarrollo y auge del neoliberalismo, las crisis financieras consecutivas de las últimas décadas o la actual crisis sanitaria global de la covid-19. A estos acontecimientos se suman procesos macrohistóricos de carácter económico como el surgimiento, desarrollo y declive de los Estados del bienestar y de la gestión keynesiana de la demanda; políticos, como la expansión de los regímenes democráticos, la política de bloques durante la Guerra Fría o el orden internacional «unimultipolar» 
de unas condiciones estructurales muy concretas que apuntalan su validez. Aspectos tan dispares como las tensiones internacionales dadas por tendencias unilaterales y excluyentes en materia de gobernanza internacional, la necesidad de ampliar el espectro semántico del trabajo ante la robotización y la incapacidad de los tejidos productivos posindustriales para garantizar el pleno empleo, el envejecimiento progresivo de la población (con la demanda asociada de cuidados de calidad $-\mathrm{y}$ remunerados ${ }^{38}-$ ) y las amenazas que se ciernen sobre la capacidad reproductiva de los seres humanos ${ }^{39}$, la situación límite a nivel ecológico, así como las posibilidades habilitadas en materia de calidad de vida por el avance tecnológico, remiten en su conjunto a las alertas planteadas por el cine, e inmediatizan la necesidad de alteraciones estructurales que definan modos no deliberadamente masculinos de organizar la experiencia comunitaria humana. En este hipotético contexto de transición, la extensa producción teórica y el prolongado activismo de las corrientes académicas y políticas feministas, pacifistas, ecologistas y antirracistas serviría, así, como guía ${ }^{40}$ a la que recurrir para la reconstrucción de las claves de funcionamiento de la especie en un esfuerzo por ahuyentar el cada vez más sólido fantasma de los hostiles escenarios de futuro que, no sin acierto, han venido vaticinando tanto el séptimo arte como la comunidad científica en su conjunto.

surgido tras el fin de esta; y demográficos, destacando en este sentido el progresivo envejecimiento de la población y la caída de las tasas de natalidad en el área eurooccidental del globo.

${ }^{38}$ El valor económico de las labores de cuidados no remuneradas alcanzaría en España hasta un $15 \%$ del PIB español (correspondiendo un 10,3\% a labores desarrolladas por mujeres y el 4,7\% restante a labores realizadas por hombres), siguiendo a la OIT (https://www.eldiario.es/economia/ trabajo-cuidados-mujeres-pib-espanol_1_2055613.html).

39 En el presente más inmediato y en los futuros imaginados ya descritos el componente reproductivo aparece como un factor fundamental. Dicho carácter nuclear de la reproducción ha llevado a expertos en demografía tales como John Maccines y Julio López Pérez a considerar dicha actividad «como un cuarto sector productivo (en realidad el primero), junto a la agricultura, la industria y los servicios» (Maccines y Pérez, 2008).

40 En su obra Masculinidades, Raewyn Connell aludía a la posibilidad real de un ordenamiento de lo sexual más ajustado a las necesidades y anhelos de la especie, enfatizando en la posibilidad concreta de nuevas políticas de lo masculino: "Creo que existe la posibilidad de nuevas políticas de masculinidad en nuevos ámbitos: por ejemplo, la política de los planes y programas de estudio, el trabajo en torno al VIH/sida y la política en contra del racismo. Creo que requerirá de nuevas formas, que incluyan a hombres y mujeres, y se centrará en el trabajo en alianzas, no tanto de "grupos de hombres». Creo que será más internacionalista que lo que la política de la masculinidad lo ha sido hasta ahora y que cuestionará la "globalización desde arriba", como otros movimientos democráticos lo han hecho. En cierto sentido se trata de una política más allá de intereses, una política de meras posibilidades. Tal vez esa sea otra forma de expresar el interés que todas las personas de este planeta tenemos en la justicia social, la paz y el equilibrio con el mundo natural» (Connell, pp. 327-328, 2003). 


\section{CONCLUSIONES}

El recorrido con perspectiva de género que aquí se ha realizado a través del cine distópico de las últimas décadas (así como las diversas críticas recogidas de tradiciones analíticas como los estudios culturales, el feminismo académico o las perspectivas ecologistas) se plantea en términos de posible diagnóstico formulado con la intención de verificar que, de cara al futuro más próximo (o al presente más inmediato, en algunos casos), los aspectos relativos al sistema sexo/género serán fundamentales en relación con el tipo de mundo que pueda esperarse que exista, así como respecto a los problemas que puedan detectarse en la práctica social actual y a las soluciones que se arbitren para lograr neutralizarlos.

Si bien la perspectiva que aquí se ha elaborado no forma parte del quehacer metodológico ortodoxo de la comunidad científica en lo que respecta a sus esfuerzos por visualizar los desafíos a que habrá de hacer frente la especie humana en momentos ulteriores de su existencia, el presente ejercicio ha tenido como objetivo poner en evidencia la relevancia del análisis feminista para una lectura del actual estado de las cosas que permita evitar los peores augurios vaticinados hasta ahora (guerras generalizadas de todo tipo, catástrofes ecológicas, infertilidad de la especie e inviabilidad de su reproducción, etc.) y ofrecer claves para afrontar el porvenir de forma proactiva y no reactiva. Se considera que esta forma crítica de abordar los retos venideros permitiría empezar a construir, desde ya, un estado de las cosas más sostenible en términos sociales, políticos y ecológicos en el que será condición sine qua non un reparto equitativo entre hombres y mujeres ${ }^{41}$ de los derechos, las obligaciones, los beneficios y las responsabilidades tanto económicas como simbólicas que se produzcan y gestionen en el seno de las sociedades.

En el proceso de investigación desarrollado, precisamente la adopción de un enfoque metodológico no ortodoxo ha funcionado como ventana de oportunidad para una visualización renovada y de mayor alcance en torno a los múltiples objetos de estudio implicados en la problemática estudiada. El recurso a fuentes documentales procedentes de diversas disciplinas, enfoques y paradigmas se considera aquí, por tanto, como una práctica científica que ha permitido la realización de un diagnóstico de mayor amplitud y agudeza (en relación con otras estrategias de análisis menos eclécticas).

Como nota final, cabría añadir que las conclusiones que del presente análisis se decantan encuentran cierto respaldo empírico en el presente histórico más inmediato. La pandemia global generada por la expansión del virus SARS-CoV-2 (más conocido por la enfermedad que produce, la covid-19) ha puesto de manifiesto cómo las colectividades humanas organizadas tienen, ante episodios de emergencia civilizatoria, su último reducto para la resistencia en los sistemas de cuidados (ya en

${ }^{41} \mathrm{Si}$ bien aquí, simplificando, se hace referencia a una ordenación binaria de lo sexual, se apunta mediante esta anotación a que un futuro justo y sostenible para la Humanidad habrá de pasar también por la inclusión de todas las identidades que no se reconocen dentro de esquemas binarios. 
su dimensión institucional -organismos administrativos asistenciales y del Estado del bienestar (instrumentos de políticas públicas que suplen la realización de labores históricamente depositadas sobre las mujeres $)^{42}-\mathrm{o}$ articulados de forma espontánea en el interior de las comunidades) ${ }^{43}$, habiéndose constituido estos en auténticos pilares para la subsistencia de los Estados durante la pandemia y el confinamiento. En este sentido, puede decirse que la actual crisis sanitaria global ha puesto de manifiesto (en consonancia con las hipótesis de trabajo desde las que se ha estructurado el presente ejercicio) que, al igual que en el cine distópico, ante situaciones limítrofes con el abismo, son las pautas de interacción basadas en la cooperación, la solidaridad y la empatía (valores completamente opuestos a los asociados a las formas de masculinidad hegemónica) las que permiten generar estrategias de resiliencia y soluciones colectivas ${ }^{44}$; del mismo modo que una gestión de situaciones de crisis que ignore estas evidencias se encontrará con enormes dificultades para lograr una salida de las mismas ${ }^{45}$.

Recibido: 26 de julio de 2021; ACEPTAdo: 16 de noviembre de 2021

42 Si bien existen fuertes críticas feministas al Estado del bienestar, dadas sus carencias a la hora de relevar a las mujeres de las cargas impuestas (y no remuneradas) por la división sexual del trabajo (Sánchez Mirá, 2016).

43 Cabe hacer referencia, en relación con esto, al contexto geográfico más inmediato: en la isla de Tenerife, durante la cuarentena decretada desde marzo de 2020, se formó la red de cuidados «Nadie sin comer en Tenerife», que garantizó de forma autogestionada la alimentación de numerosas familias a lo largo de toda la isla, con una firme vocación humanitaria y antirracista. El nacimiento y consolidación de dicha iniciativa ha sido objeto de investigación por parte del colectivo artístico «Proyecto Anti», que se encuentra actualmente en el proceso de producción de un documental sobre esta (para más información, consultar la web del colectivo: https://www.proyectoanti.com).

44 En el sentido opuesto a las determinaciones adoptadas por líderes como Donald Trump o Jair Bolsonaro, el acuerdo de reconstrucción aprobado en el marco de la Unión Europea podría señalarse como posible ejemplo de una forma de hacer política en la que el multilateralismo y la búsqueda de consenso han terminado primando frente a las antiguas y nefastas formas «deliberadamente masculinas» que aún perviven en otros espacios geopolíticos.

45 No parece casual que algunos de los países más afectados por la pandemia -tales como Rusia, EE. UU. o Brasil-estén dirigidos por administraciones abiertamente reaccionarias, desprovistas de cualquier sensibilidad feminista y que se han declarado opuestas al desarrollo y fortalecimiento de los servicios del Estado del bienestar. 


\section{REFERENCIAS}

\section{REFERENCIAS BIBLIOGRÁFICAS}

Amorós, C. (1994). Historia de la teoría feminista. Madrid: Instituto de Investigaciones Feministas.

Bauman, Z. (2017). Retrotopía. Paidós Ibérica.

Bernárdez Rodal, A. (2002). "Violencia y cine: el sabor amargo de una fascinación», en Violencia de género y sociedad: una cuestión de poder. Ayuntamiento de Madrid, pp. 87-108. ISBN 84-699-7328-2.https://eprints.ucm.es/id/eprint/10475/1/violencia_de_genero_poder.pdf.

Bourdieu, P. (2000). La dominación masculina. Editorial Anagrama.

Butler, J. (1990). El género en disputa. Paidós Ibérica.

Castells, M. (1998). «El reverdecimiento del yo: el nacimiento del movimiento ecologista». La Factoría, n. ${ }^{\circ}$ 5. Febrero-mayo. https://www.uv.mx/mie/files/2012/10/SESION4-9Sept-Reverdecimientodelyo-Castells.pdf.

Connell, R. (2003). Masculinidades. UNAM.

Corbetta, P. (2007). Metodología y técnicas de investigación social. S.A. McGraw-Hill/Interamericana de España.

DuArte, C.M. (coord.) (2006). Cambio global. Impacto de la actividad humana sobre el sistema tierra. Colección Divulgación. Consejo Superior de Investigaciones Científicas. http://aeclim.org/ wp-content/uploads/2016/01/Cambio_global.pdf.

Erreguerena Albateiro, M.J. (2008). «La distopía: una visión del futuro». Anuario de investigación. Diciembre, UAM, pp. 556-572. https://publicaciones.xoc.uam.mx/Recurso.php.

Federici, S. (2018). El patriarcado del salario. Traficantes de Sueños. https://www.traficantes.net/ sites/default/files/pdfs/TDS_map49_federici_web_0.pdf.

Fernández Calleja, J.M. (2014). «La primera guerra mundial y el modo deliberadamente masculino de hacer política». Historia y Comunicación Social, vol. 19, pp. 79-97. https://revistas. ucm.es/index.php/HICS/article/view/47286/44336.

Gabbiadini, A., Riva, P., Andrighetto, L., Volpato, C. y Bushman, B.J. (2016). «Acting like a Tough Guy: Violent-Sexist Video Games, Identification with Game Characters, Masculine Beliefs, \& Empathy for Female Violence Victims». PLoS ONE 11(4): e0152121. https:// pubmed.ncbi.nlm.nih.gov/27074057/.

García Fernando, M. et al. (2015). El análisis de la realidad social: métodos y técnicas de investigación. Alianza Editorial.

Gutiérrez de Terán, J.O. (2007). «Cine y violencia». Escuela Abierta, vol. 10, pp. 91-99. https:// ea.ceuandalucia.es/index.php/EA/article/view/99/68.

Halberstam, J. (2008). Masculinidad femenina. Egales.

Hernández Ruiz, V. (2019). «La inverosimilitud de un mundo posible sin amor. El cuento de la criada, de Margaret Atwood». Castilla. Estudios de Literatura 10 (2019): 1-22. https://dialnet.unirioja.es/descarga/articulo/7116250.pdf.

Horkheimer, M. y Adorno, T.W. (2007). Dialéctica de la Ilustración. Obra completa 3. Akal.

IACOB, M. y Rautoiu, A. (2016). «El monstruo y la censura: La superheroización del vampiro en los cómics de Marvel». En David Roas (ed.), El monstruo fantástico. Visiones y perspectivas. Aluvión Editorial, pp. 43-59. 
IAdevito, P. (2014). «Teorías de género y cine. Un aporte a los estudios de la representación». Universitas Humanistica, 78, 211-237. doi: 10.11144/Javeriana.UH78.tgcu.

Jiménez JAÉn, M. (2011). «Conocimiento social y estudios de género. Singularidades». Sistema: revista de ciencias sociales, $\mathrm{n} .^{\circ} 222$, pp. $79-94$.

Laclau, E. (2005). La razón populista. S.L Fondo de Cultura Económica de España.

Marcellesi, F. (2008). «Ecología política: génesis, teoría y praxis de la ideología verde». Cuadernos Bakeaz, n. ${ }^{\circ}$ 85, 2008, 16 pp. https://www.fuhem.es/wp-content/uploads/2019/08/ecologia_politica.pdf.

Marzábal Albaina, Í. (2009). «What future awaits us? Film and “dystopia”».J Med Mov 2009; 5(4): 123-124. https://revistas.usal.es/index.php/medicina_y_cine/article/download/13820/14264/.

Menéndez Menéndez, M. y Fernández Morales, M. (2015). «Lo heroico en el cine de masas: género y valor en Los juegos del hambre». L'Atalante Revista de estudios cinematográficos, vol. 20, pp. 92-100. http://www.revistaatalante.com/index.php?journal=atalante\&page=article\&op=view\&path\%5B\%5D=315\&path\%5B\%5D=268.

Riestra-Camacho, R. (2014): «Recodificación del género a través de la heroína distópica: Análisis de Divergente». Verbeia 2018 ISSN 2444-1333, año IV, n. ${ }^{\circ}$ 3, 118-131. https://www.ucjc. edu/wp-content/uploads/7_Rocio_Riesta_Camacho.pdf.

Veiga, F. (2015): El desequilibrio como orden. Una historia de la posguerra Fría. Alianza Editorial.

Willis, P. (2008). «Los soldados rasos de la Modernidad». Revista de Sociología de la Educación-RASE, ISSN-e 2605-1923, vol. 1, n. ${ }^{\circ}$ 3, pp. 43-66. https://ojs.uv.es/index.php/RASE/article/ view/8568/8111.

Woolf, V. (2017): Un cuarto propio/ Tres Guineas. Debolsillo.

\section{REFERENCIAS FILMOGRÁFICAS}

Carolco Pictures, Pacific Western, Lightstorm Entertainment (Productor: James Cameron) (Distribuidora: TriStar Pictures). Cameron, James (director). (1991). Terminator 2: el juicio final [cinta cinematográfica]. Estados Unidos: Pacific Western.

Co-producción Reino Unido-Japón-Estados Unidos; Universal Pictures, Strike Entertainment, Hit \& Run Productions, Toho-Towa (productores) y Cuarón. A (director). (2006). Children of men [cinta cinematográfica]. Reino Unido: Universal Pictures.

Lionsgate, Color Force (productores). Ross, Gary (director). (2012). Los juegos del hambre [cinta cinematográfica]. Estados Unidos: Lionsgate.

Netflix (productor), 2016. Black Mirror: Men Against Fire [serie de televisión]. Reino Unido.

Netflix (productor), 2017. Black Mirror: Metalhead [serie de televisión]. Reino Unido

Pacific Western, Hemdale (Distribuidora: Orion Pictures). Cameron, James (director). (1984). Terminator [cinta cinematográfica]. Estados Unidos: Pacific Western.

WARner Bros. (productora). Wachowski, Lilly y Wachowski, Lana (directoras). (2003). The Matrix Reloaded [cinta cinematográfica]. Estados Unidos: Warner Bros.

Warner Bros., Village Roadshow, Groucho Film Partnership (Productor: Joel Silver). Wachowski, Lilly y Wachowski, Lana (directoras). (1999). The Matrix. [cinta cinematográfica]. Estados Unidos: Warner Bros. 